\title{
Labyrinthe
}

$13 \mid 2002$

Numéro 13

\section{Institutions, services publics et architecture}

Institutions, services publics et architecture, colloque international organisé par l'École pratique des hautes études (IVe section, sciences historiques et philo-logiques) et la TU - Dresden. Sénat, palais du Luxembourg, 13 et 14 juin 2002.

\section{Christian Hottin}

\section{OpenEdition Journals}

Édition électronique

URL : http://journals.openedition.org/labyrinthe/1491

DOI : $10.4000 /$ labyrinthe. 1491

ISSN : 1950-6031

Éditeur

Hermann

Édition imprimée

Date de publication : 15 novembre 2002

Pagination : 140-143

Référence électronique

Christian Hottin, «Institutions, services publics et architecture », Labyrinthe [En ligne], 13 | 2002, mis en ligne le 24 février 2007, consulté le 22 septembre 2020. URL : http://journals.openedition.org/ labyrinthe/1491 ; DOI : https://doi.org/10.4000/labyrinthe.1491

Ce document a été généré automatiquement le 22 septembre 2020

Propriété intellectuelle 


\title{
Institutions, services publics et architecture
}

\author{
Institutions, services publics et architecture, colloque international \\ organisé par l'École pratique des hautes études (IVe section, sciences \\ historiques et philo-logiques) et la TU - Dresden. Sénat, palais du \\ Luxembourg, 13 et 14 juin 2002.
}

\section{Christian Hottin}

1 Deux établissements ayant opéré des rapprochements institutionnels et désormais habitués à travailler ensemble ont choisi de réunir un certain nombre de leurs membres pour un colloque qui plaçait précisément l'institutionnalité parmi ses préoccupations centrales. Au Sénat, deux journées de communications et de débats (en français et en allemand), entrecoupés de visites, ont été l'occasion d'explorer les rapports entre architecture et institutions de part et d'autre du Rhin, dans une perspective qui, tout en faisant la part belle à l'histoire de l'architecture, ne négligeait pas l'appréhension du fait architectural par d'autres disciplines (sociologie, anthropologie). Au détour des échanges, on pouvait s'en douter, ce furent les rapports particuliers des deux nations à l'institutionnalité qui se sont dévoilés.

2 En introduction du colloque, Jean-Michel Leniaud, après avoir dressé le tableau de douze années de recherches en histoire de l'architecture des XIX et $\mathrm{XX}^{\mathrm{e}}$ siècles à l'École pratique des hautes études, a cerné les outils conceptuels propres à aider la compréhension de l'ensemble des productions architecturales liées aux pouvoirs publics au cours de l'époque contemporaine : l'importance du programme défini par les instances administratives, la symbolique sous-jacente au monument, rarement exprimée ouvertement par les architectes, et enfin le "caractère » des bâtiments, terme au contraire abondamment employé et explicité tout au long de la période. Le maniement de ces outils suppose cependant la connaissance approfondie de toutes les conditions d'existence et de développement de cette architecture : cadres administratifs de création et instances de contrôle, cadres éducatifs. Quelques grands ensembles de problématiques apparaissent à la confluence de ces différents angles d'attaque: inflexions chronologiques de la production architecturale, expressions 
architecturales des tensions entre centre et périphérie, rapports entre États, divergence des choix stylistiques, traitement du décor. Sans constituer une mise en oeuvre systématique de tout ou partie de ce programme, la plupart des contributions présentées s'inscrivent dans ces axes de réflexion et de recherche.

3 Hasard ou choix délibéré lié à la localisation des séances, de nombreuses interventions portaient sur l'architecture des édifices parlementaires, domaine de la production architecturale où les liens avec les pouvoirs publics sont plus que partout ailleurs sensibles. Si l'on a pu par moment croire à un colloque consacré uniquement à ce type de constructions, d'autres communications ont contribué à équilibrer le contenu de la manifestation.

4 La place de l'enseignement, évoquée par Jean-Michel Leniaud au nombre des paramètres devant être pris en compte pour comprendre la condition de production de l'architecture publique, a été clairement illustrée par Basile Baudez : les académies en Europe aux XVIII et $\mathrm{XIX}^{\mathrm{e}}$ siècles ont souvent proposé des modèles architecturaux pour diverses sortes d'édifices publics. Quelle fut la portée effective de ces définitions de types théoriques? L'analyse comparée de deux séries d'édifices, d'une part les palais de justice et d'autre part les hôpitaux, montre les limites de la diffusion des modèles élaborés dans les écoles : si le palais de justice a en général été construit en suivant les préceptes enseignés dans les académies, l'hôpital au XIX siècle n'a que rarement l'aspect pavillonnaire préconisé par les enseignants et les auteurs des traités d'architecture.

5 Deux contributions s'attachaient à mettre en évidence l'importance des symboles dans la construction de l'institution, y compris quand ceux-ci ne sont qu'indirectement liés à l'architecture. Catherine Granger, en analysant le contenu du musée des souverains installé par Napoléon III au Louvre a mis en évidence la complexité d'une construction symbolique qui vise à légitimer une dynastie nouvelle tout en l'inscrivant dans la continuité des précédentes : les objets se rapportant à la famille Bonaparte, qui sont de loin les plus nombreux, voisinent avec le portrait de Jean le Bon et des reliques de la captivité de Saint Louis. Dans l'architecture universitaire française, les symboles présents dans le décor sculpté qui orne les façades sont également une occasion pour les institutions de représenter leur identité. Bien loin de représenter uniquement les disciplines enseignées au sein de l'établissement, ces sculptures doivent être regardées comme des images de valeur du groupe humain qui gravite autour de l'établissement, telles sont en tout cas les fonctions du bélier, de la chouette et de l'abeille à l'École polytechnique et à l'École centrale. Dans cet exposé, Christian Hottin s'est également attaché à montrer que les pouvoirs publics sont paradoxalement peu présents dans les représentations figurées de ces établissements sur lesquels ils exercent pourtant leur tutelle et qu'ils contribuent à reconstruire ou embellir.

6 Trois interventions avaient pour but de préciser l'évolution architecturale de types de bâtiments particuliers, soit que l'on se situe dans le cadre d'un long xix ${ }^{e}$ siècle, soit au contraire que l'on choisisse de montrer les répercussions d'un événement historique majeur sur le plan architectural. Ce fut le choix de Nicolas Padiou, avec une contribution à l'étude de l'architecture cultuelle dans le département de Meurthe-etMoselle pendant et après la Grande Guerre.

7 Hans-Georg Lippert et Marie Gloc-Dechezleprestre quant à eux s'attachèrent à définir l'évolution de l'architecture des universités en Allemagne et des hôtels de ville en France entre 1800 et 1914. Dans le premier cas, on assiste à une évolution linéaire, et 
essentiellement stylistique pendant une grande partie de la période considérée. Malgré l'influence du cours professé par J.-N. Durand à l'École polytechnique, les architectes allemands adoptent pour modèle des universités construites dans la première moitié du $\mathrm{XIX}^{\mathrm{e}}$ siècle les palais du baroque tardif $\mathrm{du}$ siècle précédent. Après 1850 , le néorenaissance apparaît comme le langage quasi universel de l'architecture officielle allemande, et les universités ne font pas exception à cette règle. Enfin, l'avènement du Reich et la grande prospérité de la fin du siècle donnent naissance à des édifices gigantesques, dont l'université de Strasbourg est un des meilleurs exemples. Ces constructions colossales mais difficilement adaptables aux besoins spécifiques des différentes disciplines semblent bien vite inadaptées : alors apparaissent les pavillons des premiers campus.

8 L'architecture de l'hôtel de ville en France connaît à la même époque un cheminement plus complexe que ne l'ont laissé supposer les études menées jusqu'à présent. Sans nier la réalité et l'importance quantitative des mairies "républicaines» étudiées par Maurice Agulhon, il faut nuancer cette présentation : le contexte local, la présence du bâti existant, la lenteur des réalisations ont abouti à des situations très diverses qu'illustrent aussi bien la mairie Art nouveau d'Euville (Meuse), unique en France, que l'hôtel de ville néo-classique de Moulins.

9 C'est autour de l'architecture des édifices parlementaires que le débat a été le plus riche. C'est également au cours de cette partie du colloque qu'ont été présentées les seules interventions offrant des études comparatives entre institutions françaises et allemandes.

10 Grâce à Jacques Patureau, architecte en charge du Sénat, la structure composite de cet édifice aux multiples strates est désormais bien connue : sans la superposer totalement à l'évolution de l'institution aux multiples noms qui est abritée par le palais du Luxembourg, on peut esquisser un parallèle entre transformations architecturales et mutations institutionnelles. Les mutations successives de l'institution transforment la physionomie intérieure de l'édifice, obligeant les architectes à installer des salles de séances toujours plus vastes, tout d'abord sans modifier l'aspect extérieur du bâtiment, puis en lui donnant une extension plus importante. À cette histoire complexe et quelque peu chaotique, on peut opposer celle du parlement de Vienne, construit en style néoclassique d'une grande unité par Théophil Hansen, dont l'histoire a été retracée par Jacques Le Ridder.

11 Une approche plus directement comparative a été fournie par Markus Dauss avec l'analyse de la constitution du Palais-Bourbon et du Reichstag en tant que lieux de mémoire nationaux. Les oppositions architecturales évoquées pour le Sénat français et le Parlement autrichien se retrouvent ici : en France, un édifice maintes fois transformé, en Allemagne une construction décidée et exécutée en une seule fois sous l'Empire. Pourtant, tout au long du siècle et en dépit des changement politiques, le Palais Bourbon donne lieu à des lectures homogènes, ce qui n'est pas le cas pour le Reichstag, dont l'éclectisme et l'historicisme traduisent les tensions d'un grande nation récemment unifiée.

Une des plus grandes qualités du colloque est sans doute de n'avoir pas réduit l'étude des édifices parlementaires à leur seule enveloppe architecturale, mais d'avoir tenter une analyse, incluant la dimension architecturale, des rapports entre institutions représentatives et populations représentées. Pour l'Assemblée nationale et le Bundestag, Alexander Weiss a montré les transformations induites par le 
développement des masses-médias, tandis que Romy Messerschmidt a étudié les rapports entre représentants et représentés dans les institutions parlementaire dans la perspective de la théorie institutionnelle : quel rôle joue la structuration de l'espace parlementaire dans la légitimation d'un parlement? Quelques études de cas (le palais de la République à Berlin Est, le parlement d'Ottawa et le Palais Bourbon à Paris) apportent des éléments de réponse.

13 Karl-Siegbert Rehberg a apporté la conclusion de ces deux journées de travaux en évoquant les nombreuses « constructions institutionnelles » qui avaient été étudiées : toutes concourent à montrer que la stabilisation institutionnelle des relations sociales repose avant tout sur la possibilité de rendre visibles des représentations de l'ordre et des prétentions d'autorité, ce qui contribue à produire une « croyance à la légitimité ». 